\title{
Existence results for fractional hybrid differential systems in Banach algebras
}

\section{Tahereh Bashiri' ${ }^{1}$, Seiyed Mansour Vaezpour ${ }^{1}$ and Choonkil Park ${ }^{2 *}$}

\author{
Correspondence: \\ baak@hanyang.ac.kr \\ ${ }^{2}$ Research Institute for Natural \\ Sciences, Hanyang University, Seoul, \\ 133-791, Korea \\ Full list of author information is \\ available at the end of the article
}

\begin{abstract}
In this manuscript we investigate the existence of solutions for the following system of fractional hybrid differential equations (FHDEs):

$$
\begin{cases}D^{p}\left[\frac{\theta(t)-w(t, \theta(t))}{u(t, \theta(t))}\right]=v(t, \vartheta(t)), & t \in J, \\ D^{p}\left[\frac{\vartheta(t)-w(t, \vartheta(t))}{u(t, \vartheta(t))}\right]=v(t, \theta(t)), & t \in J, 0<p<1, \\ \theta(0)=0, \quad \vartheta(0)=0, & \end{cases}
$$

where $D^{r}$ denotes the Riemann-Liouville fractional derivative of order $r, J=[0,1]$, and the functions $u: J \times \mathbb{R} \rightarrow \mathbb{R} \backslash\{0\}, w: J \times \mathbb{R} \rightarrow \mathbb{R}, w(0,0)=0$ and $v: J \times \mathbb{R} \rightarrow \mathbb{R}$ satisfy certain conditions.

Here, we extend the Dhage hybrid fixed point theorem (Dhage in Kyungpook Math. J. 44:145-155, 2004) and then present some results on the existence of coupled fixed points for a category of operators in Banach algebra. Also, an example is analyzed to show the use of the reported results.
\end{abstract}

MSC: $34 \mathrm{~A} 38 ; 32 \mathrm{~A} 65 ; 47 \mathrm{H} 10 ; 26 \mathrm{~A} 33$

Keywords: hybrid initial value problem; Banach algebras; coupled fixed point theorem; Riemann-Liouville fractional derivative

\section{Introduction}

It is worth noting that the perturbation techniques are useful in the nonlinear analysis for studying the dynamical systems described by nonlinear differential and integral equations. Some differential equations representing a certain dynamical system have not analytical solution, so the perturbation of such problems can be helpful. The perturbed differential equations are categorized into various types. An important type of these perturbations is called a hybrid differential equation (i.e. quadratic perturbation of a nonlinear differential equation). See for more details [2] and the references therein.

Recently, this issue has received much attention [3-6]. We mention that the hybrid fixed point theory can be used to develop the existence theory for the hybrid equations. For more details we refer the reader to [7-10]. Dhage and Lakshmikantham [8] scrutinized

(c) 2016 Bashiri et al. This article is distributed under the terms of the Creative Commons Attribution 4.0 International License (http://creativecommons.org/licenses/by/4.0/), which permits unrestricted use, distribution, and reproduction in any medium, provided you give appropriate credit to the original author(s) and the source, provide a link to the Creative Commons license, and indicate if changes were made. 
the following first order hybrid differential equation:

$$
\left\{\begin{array}{l}
\frac{d}{d t}\left[\frac{\theta(t)}{u(t, \theta(t))}\right]=v(t, \theta(t)), \quad \text { a.e. } t \in J, \\
\theta\left(t_{0}\right)=\theta_{0} \in \mathbb{R},
\end{array}\right.
$$

where $J=[0, T), u \in C(J \times \mathbb{R}, \mathbb{R} \backslash\{0\})$, and $v \in \mathcal{C}(J \times \mathbb{R}, \mathbb{R})$. They were the first authors able to prove the existence and uniqueness results as well as various fundamental differential inequalities corresponding to the hybrid differential equations. In fact, with the help of the theory of inequalities, the existence of extremal solutions and a comparison result were proved by Dhage and Lakshmikantham.

The topic of fractional calculus which deals with derivatives and integrals of arbitrary orders started to be used to model successfully several physical phenomena [11-13]. Evidently, this branch of calculus has found numerous miscellaneous applications connected with real world problems as they appear in many fields of science and engineering, including fluid flow, signal and image processing, fractals theory, control theory, electromagnetic theory, fitting of experimental data, optics, potential theory, biology, chemistry, diffusion, and viscoelasticity. For some recent developments on the topic, see [14-19].

Zhao et al. [20] introduced FHDEs. They took the initiative by regarding the FHDE with $\mathrm{R}-\mathrm{L}$ differential operators

$$
\left\{\begin{array}{l}
D^{q}\left[\frac{\theta(t)}{u(t, \theta(t))}\right]=v(t, \theta(t)), \quad \text { a.e. } t \in J, 0<q<1, \\
\theta\left(t_{0}\right)=\theta_{0} \in \mathbb{R},
\end{array}\right.
$$

where $J=[0, T), u \in C(J \times \mathbb{R}, \mathbb{R} \backslash\{0\})$, and $v \in \mathcal{C}(J \times \mathbb{R}, \mathbb{R})$. Beside that, by using the mixed Lipschitz and Carathéodory conditions they proved the existence theorem for FHDEs.

Sitho et al. [21] studied existence results for the initial value problems of hybrid fractional integro-differential equations:

$$
\left\{\begin{array}{l}
D^{q}\left[\frac{\theta(t)-\sum_{i=1}^{m} I^{\beta_{i}} w_{i}(t, \theta(t))}{u(t, \theta(t))}\right]=v(t, \theta(t)), \quad \text { a.e. } t \in J, \\
\theta(0)=0 \in \mathbb{R},
\end{array}\right.
$$

where $D^{q}$ denotes the $\mathrm{R}-\mathrm{L}$ fractional derivative of order $q, 0<\alpha \leq 1, I^{\phi}$ is the $\mathrm{R}$ - $\mathrm{L}$ fractional integral of order $\phi>0, \phi \in\left\{\beta_{1}, \beta_{2}, \ldots, \beta_{m}\right\}, J=[0, T), u \in C(J \times \mathbb{R}, \mathbb{R} \backslash\{0\})$, and $v, w \in$ $\mathcal{C}(J \times \mathbb{R}, \mathbb{R})$ with $w(0,0)=0$.

The research of coupled systems within fractional differential equations become interesting, mainly because these kinds of research appear in various problems, e.g. see [22-25]. Recently, Su [26] analyzed a two-point boundary value problem corresponding to a coupled system of fractional differential equations. Also, Gafiychuk et al. [27] discussed the solutions of particular case, namely the coupled nonlinear fractional reaction-diffusion equations.

Also, in 2014, Bashir Ahmad et al. [28] considered the following Dirichlet boundary value problem of coupled hybrid fractional differential equations:

$$
\left\{\begin{array}{l}
{ }^{c} D^{\delta}\left[\frac{x(t)}{f_{1}(t, x(t), y(t))}\right]=h_{1}(t, x(t), y(t)), \quad t \in J, 1<\delta \leq 2, \\
D^{\gamma}\left[\frac{y(t)}{f_{2}(t, x(t), y(t))}\right]=h_{2}(t, x(t), y(t)), \quad t \in J, 1<\gamma \leq 2, \\
x(0)=x(1)=0, \quad y(0)=y(1)=0,
\end{array}\right.
$$


where $f_{i} \in C(J \times \mathbb{R} \times \mathbb{R}, \mathbb{R} \backslash\{0\})$ and $h_{i} \in C(J \times \mathbb{R} \times \mathbb{R}, \mathbb{R}), i=1,2$.

Here, we are studying the existence of solutions for the system of fractional hybrid differential equations

$$
\begin{cases}D^{p}\left[\frac{\theta(t)-w(t, \theta(t))}{u(t, \theta(t))}\right]=v(t, \vartheta(t)), & t \in J, \\ D^{p}\left[\frac{\vartheta(t)-(t, \vartheta(t))}{u(t, \vartheta(t))}\right]=v(t, \theta(t)), & t \in J, 0<p<1, \\ \theta(0)=0, \quad \vartheta(0)=0 . & \end{cases}
$$

First of all, we prove a coupled fixed point theorem which is a generalization of a fixed point theorem of Dhage [1] in Banach algebras.

\section{Preliminaries}

Let $C(J \times \mathbb{R}, \mathbb{R})$ be the category of continuous functions $u: J \times \mathbb{R} \rightarrow \mathbb{R}$.

Definition 1 ([29]) The form of the Riemann-Liouville fractional integral operator of or$\operatorname{der} \sigma>0$ of the function $w \in L^{1}\left(\mathbb{R}^{+}\right)$is defined as

$$
I^{\sigma} w(t)=\frac{1}{\Gamma(\sigma)} \int_{0}^{t}(t-l)^{\sigma-1} w(l) d l
$$

Definition 2 ([29]) The Riemann-Liouville fractional derivative of order $\sigma>0$ of a continuous function $w:(0, \infty) \rightarrow \mathbb{R}$ is

$$
D^{\sigma} w(t)=\frac{1}{\Gamma(n-\sigma)}\left(\frac{d}{d t}\right)^{n} \int_{0}^{t}(t-l)^{n-\sigma-1} w(l) d l
$$

where $n=[\sigma]+1$.

Lemma $1([29,30])$ Let us consider $0<\sigma<1$ and $w \in L^{1}(0,1)$. Then $D^{\sigma} I^{\sigma} w(t)=w(t)$,

$$
I^{\sigma} D^{\sigma} w(t)=w(t)-\frac{\left[D^{\sigma-1} w(t)\right]_{t=0}}{\Gamma(\sigma)} t^{\sigma-1}
$$

hold almost everywhere on $J$.

What follows next is a fixed point theorem in Banach algebras due to Dhage ([1]).

Lemma 2 ([1]) Let $S$ be a non-empty, closed, convex, and bounded subset of a Banach algebra $\Im$ and let $E, G: \Im \rightarrow \Im$ and $F: S \rightarrow \Im$ be two operators fulfilling the following properties:
(a) E and $G$ are Lipschitzian with Lipschitz constants $\sigma$ and $\delta$, respectively,
(b) $F$ is completely continuous,
(c) $\theta=E \theta F \vartheta+G \theta \Rightarrow \theta \in S$ for all $\vartheta \in S$, and
(d) $\sigma \digamma+\delta<1$, where $\digamma=\|F S\|=\sup \{\|F(\theta)\|: \theta \in S\}$.

Then the operator equation $E \theta F \theta+G \theta=\theta$ has a solution in $S$.

Below we recall the definition of a coupled fixed point for a bivariate mapping. 
Definition 3 ([31]) An element $(\theta, \vartheta) \in \mathfrak{I} \times \mathfrak{\Im}$ is called a coupled fixed point of a mapping $T: \Im \times \Im \rightarrow \Im$ if $T(\theta, \vartheta)=\theta$ and $T(\vartheta, \theta)=\vartheta$.

By a solution of the FHDEs system (1) we mean a function $(\theta, \vartheta) \in \operatorname{AC}(J, \mathbb{R} \times \mathbb{R})$ with the following properties:

(i) the function $t \rightarrow \frac{\theta-w(t, \theta)}{u(t, \theta)}$ is absolutely continuous for each $\theta \in \mathbb{R}$, and

(ii) $(\theta, \vartheta)$ satisfies the system of equations in (1),

where $\mathrm{AC}(J, \mathbb{R} \times \mathbb{R})$ is the space of absolutely continuous real-valued functions defined on $J$.

\section{Fixed point theorems}

Throughout this section, let $\mathfrak{I}=C(J, \mathbb{R})$ be equipped with the supremum norm. Clearly it is a Banach algebra with respect to pointwise operations and the supremum norm.

Define scalar multiplication and the sum on $\mathfrak{\Im} \times \mathfrak{\Im}$ as follows:

$$
\left(\theta_{1}, \vartheta_{1}\right)+\left(\theta_{2}, \vartheta_{2}\right)=\left(\theta_{1}+\theta_{2}, \vartheta_{1}+\vartheta_{2}\right)
$$

and

$$
\mu(\theta, \vartheta)=(\mu \theta, \mu \vartheta)
$$

for $\mu \in \mathbb{R}$. Then $\Im \times \Im$ is a vector space on $\mathbb{R}$.

In the following lemma we introduce a certain Banach algebra which is used in proving our results.

Lemma 3 Let $\widetilde{\Im}:=\Im \times \Im$. Define the product

$$
(\theta, \vartheta)\left(\theta^{\prime}, \vartheta^{\prime}\right)=\left(\theta \theta^{\prime}, \vartheta \vartheta^{\prime}\right)
$$

and

$$
\|(\theta, \vartheta)\|=2(\|\theta\|+\|\vartheta\|) .
$$

Then $\widetilde{\Im}$ is a Banach algebra with respect to the above norm and multiplication.

Proof By inspection $\widetilde{\Im}$ is an algebra and $\|\cdot\|$ is a norm on $\widetilde{\Im}$. We notice that

$$
\begin{aligned}
\left\|\left(\theta_{1}, \theta_{2}\right)\left(\theta_{1}^{\prime}, \theta_{2}^{\prime}\right)\right\| & =\left\|\left(\theta_{1} \theta_{1}^{\prime}, \theta_{2} \theta_{2}^{\prime}\right)\right\| \\
& =2\left(\left\|\theta_{1} \theta_{1}^{\prime}\right\|+\left\|\theta_{2} \theta_{2}^{\prime}\right\|\right) \\
& \leq 2\left(\left\|\theta_{1}\right\|\left\|\theta_{1}^{\prime}\right\|+\left\|\theta_{2}\right\|\left\|\theta_{2}^{\prime}\right\|\right) \\
& \leq 2\left(\left\|\theta_{1}\right\|\left(\left\|\theta_{1}^{\prime}\right\|+\left\|\theta_{2}^{\prime}\right\|\right)+\left(\left\|\theta_{1}\right\|+\left\|\theta_{2}\right\|\right)\left\|\theta_{2}^{\prime}\right\|\right) \\
& \leq 2\left[\left(\left\|\theta_{1}\right\|+\left\|\theta_{2}\right\|\right)\left(\left\|\theta_{1}^{\prime}\right\|+\left\|\theta_{2}^{\prime}\right\|\right)+\left(\left\|\theta_{1}\right\|+\left\|\theta_{2}\right\|\right)\left(\left\|\theta_{1}^{\prime}\right\|+\left\|\theta_{2}^{\prime}\right\|\right)\right] \\
& \leq 2\left(2\left(\left\|\theta_{1}\right\|+\left\|\theta_{2}\right\|\right)\left(\left\|\theta_{1}^{\prime}\right\|+\left\|\theta_{2}^{\prime}\right\|\right)\right) \\
& \leq\left(\left\|\left(\theta_{1}, \theta_{2}\right)\right\|\right)\left(\left\|\left(\theta_{1}^{\prime}, \theta_{2}^{\prime}\right)\right\|\right),
\end{aligned}
$$

so $\|\cdot\|$ is an algebra norm on $\widetilde{\Im}$. 
Now, we prove a coupled fixed point theorem, which is a generalization of Lemma 2 of Dhage [1].

Let us denote by $\Phi$ the family of all functions $\varphi: \mathbb{R}^{+} \rightarrow \mathbb{R}^{+}$fulfilling $\varphi(r)<r$ for $r>0$ and $\varphi(0)=0$.

Definition 4 Let $\Im$ be a Banach space. An operator $C: \mathfrak{\Im} \rightarrow \Im$ is called $\sigma$-nonlinear contraction if there exist a real constant $\sigma \in(0,1)$ and a function $\varphi_{C} \in \Phi$ such that

$$
\|C \theta-C \vartheta\| \leq \sigma \varphi_{C}(\|\theta-\vartheta\|)
$$

for all $\theta, \vartheta \in \mathfrak{I}$. We call the function $\varphi_{C}$ a nonlinear function of $C$ on $X$.

Theorem 1 Let $S$ be a non-empty, closed, convex, and bounded subset of the Banach algebra $\Im$ and $\widetilde{S}=S \times S$. Suppose that $E, G: \Im \rightarrow \Im$ and $F: S \rightarrow \Im$ be three operators such that

(C1) The operators $E$ is $\sigma$-nonlinear contraction with nonlinear contraction function $\varphi_{E}$, and $G$ is $\delta$-nonlinear contraction with nonlinear contraction function $\varphi_{G}$,

(C2) $F$ is completely continuous,

(C3) $\theta=E \theta F \vartheta+G \theta \Rightarrow \theta \in S$ for all $\vartheta \in S$, and

(C4) $4 \sigma\|F S\|+\delta<1$, where $\|F S\|=\sup \{\|F(x)\|: x \in S\}$.

Then the operator $T(\theta, \vartheta)=E \theta F \vartheta+G \theta$ has at least a coupled fixed point in $\widetilde{S}$.

Proof By inspection we conclude that $\widetilde{S}$ is a non-empty, closed, convex, and bounded subset of the Banach algebra $\widetilde{I}$. Define $\widetilde{E}, \widetilde{G}: \widetilde{\Im} \rightarrow \widetilde{\Im}$, and $\widetilde{F}: \widetilde{S} \rightarrow \widetilde{\Im}$ by

$$
\begin{aligned}
& \widetilde{E}(\theta, \vartheta)=(E \theta, E \vartheta), \\
& \widetilde{F}(\theta, \vartheta)=(F \vartheta, F \theta), \\
& \widetilde{G}(\theta, \vartheta)=(G \theta, G \vartheta) .
\end{aligned}
$$

It is sufficient to prove $\widetilde{E}(\theta, y \vartheta) \widetilde{F}(\theta, \vartheta)+\widetilde{G}(\theta, \vartheta)=(\theta, \vartheta)$ has at least one solution, because

$$
\begin{aligned}
(T(\theta, \vartheta), T(\vartheta, \theta)) & =(E \theta B \vartheta+C \theta, E \vartheta F \theta+G \vartheta) \\
& =(E \theta, E \vartheta)(F \vartheta, F \theta)+(G \theta, G \vartheta) \\
& =\widetilde{E}(\theta, \vartheta) \widetilde{F}(\theta, \vartheta)+\widetilde{G}(\theta, \vartheta)=(\theta, \vartheta),
\end{aligned}
$$

which implies that $T(\theta, \vartheta)$ has at least one coupled fixed point. We claim that the operators $\widetilde{E}, \widetilde{F}$, and $\widetilde{G}$ satisfy all the conditions of Lemma 2 on the Banach algebra $\widetilde{\Im}$. First, we show that $\widetilde{E}$ and $\widetilde{G}$ are Lipschitzian. By using the condition $(\mathrm{C} 1)$, for every $\theta=\left(\theta_{1}, \theta_{2}\right)$, $\vartheta=\left(\vartheta_{1}, \vartheta_{2}\right) \in \widetilde{\Im}$ we obtain

$$
\begin{aligned}
\|\widetilde{E} \theta-\widetilde{E} \vartheta\| & =\left\|\left(E \theta_{1}, E \theta_{2}\right)-\left(E \vartheta_{1}, E \vartheta_{2}\right)\right\| \\
& =\left\|\left(E \theta_{1}-E \vartheta_{1}, E \theta_{2}-E \vartheta_{2}\right)\right\| \\
& =2\left(\left\|E \theta_{1}-E \vartheta_{1}\right\|+\left\|E \theta_{2}-E \vartheta_{2}\right\|\right) \\
& \leq 2\left(\sigma \varphi_{E}\left(\left\|\theta_{1}-\vartheta_{1}\right\|\right)+\sigma \varphi_{E}\left(\left\|\theta_{2}-\vartheta_{2}\right\|\right)\right)
\end{aligned}
$$




$$
\begin{aligned}
& <2 \sigma\left(\left\|\theta_{1}-\vartheta_{1}\right\|+\left\|\theta_{2}-\vartheta_{2}\right\|\right) \\
& =\sigma\left\|\left(\theta_{1}-\vartheta_{1}, \theta_{2}-\vartheta_{2}\right)\right\| \\
& =\sigma\|\theta-\vartheta\|,
\end{aligned}
$$

which implies that $\widetilde{E}$ is Lipschitzian with constant $\sigma$. Similarly $\widetilde{G}$ is Lipschitzian with constant $\delta$. The next step is to prove that $\widetilde{F}$ is a compact and continuous operator on $\widetilde{S}$.

Let $\left(\theta_{n}\right)=\left(\theta_{1 n}, \theta_{2 n}\right)$ be a sequence in $\widetilde{S}$ converging to a point $\theta=\left(\theta_{1}, \theta_{2}\right) \in \widetilde{S}$. Since $F$ is continuous we have

$$
\begin{aligned}
\lim _{n \rightarrow \infty} \widetilde{F} \theta_{n} & =\left(\lim _{n \rightarrow \infty} F \theta_{2 n}, \lim _{n \rightarrow \infty} F \theta_{1 n}\right) \\
& =\left(F \theta_{2}, F \theta_{1}\right)=\widetilde{F}\left(\theta_{1}, \theta_{2}\right)=\widetilde{F} \theta,
\end{aligned}
$$

so $\widetilde{F}$ is continuous.

Let $\theta=\left(\theta_{1}, \theta_{2}\right) \in \widetilde{S}$. Then we have

$$
\begin{aligned}
\left\|\widetilde{F}\left(\theta_{1}, \theta_{2}\right)\right\| & =\left\|\left(F \theta_{1}, F \theta_{2}\right)\right\| \\
& =2\left(\left\|F \theta_{1}\right\|+\left\|F \theta_{2}\right\|\right) \\
& \leq 4\|F S\|,
\end{aligned}
$$

for all $\theta \in \widetilde{S}$. This result shows that $\widetilde{F}$ is uniformly bounded on $\widetilde{S}$.

Let $\varepsilon>0$, since $F(S)$ is an equi-continuous set in $\Im$, there exists $\delta>0$ such that for $t_{1}, t_{2} \in$ $J,\left|t_{1}-t_{2}\right|<\delta$ implies that $\left|F \theta\left(t_{1}\right)-F \theta\left(t_{2}\right)\right|<\varepsilon$ for all $\theta \in S$. Then, for any $\theta=\left(\theta_{1}, \theta_{2}\right) \in \widetilde{S}$, we have

$$
\begin{aligned}
\left|\widetilde{F} \theta\left(t_{1}\right)-\widetilde{F} \theta\left(t_{2}\right)\right| & =\left|\left(F \theta_{2}\left(t_{1}\right), F \theta_{1}\left(t_{1}\right)\right)-\left(F \theta_{2}\left(t_{2}\right), F \theta_{1}\left(t_{2}\right)\right)\right| \\
& =\left|\left(F \theta_{2}\left(t_{1}\right)-F \theta_{2}\left(t_{2}\right), F \theta_{1}\left(t_{1}\right)-F \theta_{1}\left(t_{2}\right)\right)\right| \\
& =\sqrt{\left(F \theta_{1}\left(t_{1}\right)-F \theta_{1}\left(t_{2}\right)\right)^{2}+\left(F \theta_{2}\left(t_{1}\right)-F \theta_{2}\left(t_{2}\right)\right)^{2}} \\
& <\sqrt{2} \varepsilon,
\end{aligned}
$$

so $\widetilde{F}(\widetilde{S})$ is an equi-continuous set in $\widetilde{\Im}$. Thus, $\widetilde{F}(\widetilde{S})$ is compact according to the ArzeláAscoli theorem. As a result, $\widetilde{F}$ is a continuous and compact operator on $\widetilde{S}$. So, $\widetilde{F}$ is completely continuous on $\widetilde{S}$.

We show now that the hypothesis (iii) of Lemma 2 is satisfied. Let $\theta=\left(\theta_{1}, \theta_{2}\right) \in \widetilde{\Im}, \vartheta=$ $\left(\vartheta_{1}, \vartheta_{2}\right) \in \widetilde{S}$ such that $\theta=\widetilde{E} \theta \widetilde{F} \vartheta+\widetilde{G} \theta$. Then

$$
\begin{aligned}
\left(\theta_{1}, \theta_{2}\right) & =\widetilde{E}\left(\theta_{1}, \theta_{2}\right) \widetilde{F}\left(\vartheta_{1}, \vartheta_{2}\right)+\widetilde{G}\left(\theta_{1}, \theta_{2}\right) \\
& =\left(E \theta_{1}, E \theta_{2}\right)\left(F \vartheta_{2}, F \vartheta_{1}\right)+\left(G \theta_{1}, G \theta_{2}\right) \\
& =\left(E \theta_{1} F \vartheta_{2}+G \theta_{1}, E \theta_{2} F \vartheta_{1}+G \theta_{2}\right),
\end{aligned}
$$

which implies that

$$
\theta_{1}=E \theta_{1} F \vartheta_{2}+G \theta_{1}
$$




$$
\theta_{2}=E \theta_{2} F \vartheta_{1}+G \theta_{2}
$$

So, by assumption (C3), we conclude that $\theta_{1}, \theta_{2} \in S$, then $\theta \in \widetilde{S}$. Finally, we prove that $\sigma \digamma+\delta<1$, where $\digamma=\|\widetilde{F} \widetilde{S}\|=\sup \{\|\widetilde{F}(\theta)\|: \theta \in \widetilde{S}\}$. By using the assumption (C4) we obtain

$$
\begin{aligned}
\sigma \digamma+\delta & =\sigma\|\widetilde{F} \widetilde{S}\|+\delta \\
& =\sigma(2(\|F S\|+\|F S\|))+\delta \\
& =4 \sigma\|F S\|+\delta<1 .
\end{aligned}
$$

So, all conditions of Lemma 2 are satisfied and hence the operator equation $\widetilde{E} u \widetilde{F} u+\widetilde{G} u=u$ has at least one solution on $\widetilde{S}$. As a result, $T(\theta, \vartheta)$ has at least one coupled fixed point and the proof is completed.

Corollary 1 Let $S$ be a non-empty, closed, convex, and bounded subset of the Banach algebra $\Im$ and $\widetilde{S}=S \times S$. Suppose that $A, C: \Im \rightarrow \Im$ and $B: S \rightarrow \Im$ are three operators such that

(C1) E and $G$ are Lipschitzian with Lipschitz constants $\sigma$ and $\delta$, respectively,

(C2) $F$ is completely continuous,

(C3) $\theta=A \theta B \vartheta+C \theta \Rightarrow \theta \in S$ for all $\vartheta \in S$, and

(C4) $4 \sigma\|F S\|+\delta<1$, where $\|F S\|=\sup \{\|F(\theta)\|: \theta \in S\}$.

Then the operator $T(\theta, \vartheta)=E \theta F \vartheta+G \theta$ has at least a coupled fixed point in $\widetilde{S}$.

Taking $G \equiv 0$ in Theorem 1, we obtain the following.

Corollary 2 Let $S$ be a non-empty, closed, convex, and bounded subset of the Banach algebra $\Im$ and $\widetilde{S}=S \times S$. Suppose that $E: \Im \rightarrow \Im$ and $F: S \rightarrow \Im$ be two operators such that

(a) there exists $\varphi_{E} \in \Phi$ such that for all $\theta, \vartheta \in \mathfrak{I}$, we have

$$
\|E \theta-E \vartheta\| \leq \sigma \varphi_{E}(\|\theta-\vartheta\|)
$$

for some constant $\sigma>0$,

(b) $F$ is completely continuous,

(c) $\theta=E \theta F \vartheta \Rightarrow \theta \in S$ for all $\vartheta \in S$, and

(d) $4 \sigma\|F S\|=\sup \{\|F(\theta)\|: \theta \in S\}<1$.

Then the operator $T(\theta, \vartheta)=E \theta F \vartheta$ has at least a coupled fixed point in $\widetilde{S}$.

Taking $E \equiv 1$ in Theorem 1, we obtain the following.

Corollary 3 Let S be a non-empty, closed, convex, and bounded subset of the Banach space $\Im$ and $\widetilde{S}=S \times S$. Suppose that $G: \Im \rightarrow \Im$ and $F: S \rightarrow \Im$ are two operators such that

(a) there exists $\varphi_{C} \in \Phi$ such that for all $\theta, \vartheta \in \Im$, we have

$$
\|G \theta-G \vartheta\| \leq \delta \varphi_{G}(\|\theta-\vartheta\|),
$$

for some constant $\delta>0$,

(b) $F$ is completely continuous, 
(c) $\theta=G \theta+B \vartheta \Rightarrow \theta \in S$ for all $\vartheta \in S$.

Then the operator $T(\theta, \vartheta)=G \theta+F \vartheta$ has at least a coupled fixed point in $\widetilde{S}$ whenever $\delta<1$.

\section{Systems of fractional hybrid differential equations}

By applying Theorem 1, we study the existence of a solution for the FHDEs system (1) under the following general assumptions:

(H1) The functions $u: J \times \mathbb{R} \rightarrow \mathbb{R} \backslash\{0\}, w: J \times \mathbb{R} \rightarrow \mathbb{R}, w(0,0)=0$ are continuous and there exist two functions $\varphi$ and $\psi$ with bounds $\|\varphi\|$ and $\|\psi\|$, respectively, such that for all $\theta, \vartheta \in \Im$ and $t \in J$ we have

$$
|u(t, \theta(t))-u(t, \vartheta(t))| \leq \varphi(t)|\theta(t)-\vartheta(t)|
$$

and

$$
|w(t, \theta(t))-w(t, \vartheta(t))| \leq \psi(t)|\theta(t)-\vartheta(t)|
$$

(H2) There exists a continuous function $v \in C(J, \mathbb{R})$ such that

$$
v(t, \theta) \leq v(t), \quad t \in J,
$$

for all $\theta \in \mathbb{R}$, and $\frac{4}{\Gamma(p+1)}\|\varphi\|\|v\|+\|\psi\|<1$.

As a consequence of Lemma 1 we have the following result, which is useful in proving the existence results.

Lemma 4 Assume that $v \in C[0,1], 0<p<1$, and $u, w \in C([0,1] \times \mathbb{R}, \mathbb{R})$, such that $u(0,0) \neq$ 0 and $w(0,0)=0$. Then the unique solution of the following initial value problem:

$$
\left\{\begin{array}{l}
D^{p}\left[\frac{\theta(t)-w(t, \theta(t))}{u(t, \theta(t))}\right]=v(t), \quad t \in(0,1) \\
\theta(0)=0
\end{array}\right.
$$

is

$$
\theta(t)=\frac{u(t, \theta(t))}{\Gamma(p)} \int_{0}^{t} \frac{v(s)}{(t-s)^{1-p}} d s+w(t, \theta(t)), \quad t \in[0,1]
$$

Proof Let $\theta(t)$ be a solution of the problem (2). By applying to both sides of (2) the operator $I^{p}$, we get

$$
I^{p} D^{p}\left[\frac{\theta(t)-w(t, \theta(t))}{u(t, \theta(t))}\right]=I^{p} v(t)
$$

so, from Lemma 1 we conclude that

$$
\frac{\theta(t)-w(t, \theta(t))}{u(t, \theta(t))}-\frac{\left.D^{p-1}\left[\frac{\theta(t)-w(t, \theta(t))}{u(t, \theta(t))}\right]\right|_{t=0}}{\Gamma(p)} t^{p-1}=I^{p} v(t) .
$$


Since $\left.\frac{\theta(t)-w(t, \theta(t))}{u(t, \theta(t))}\right|_{t=0}=\frac{\theta(0)-w(0,0)}{u(0, \theta(0))}=\frac{0}{u(0,0)}=0$ (due to the fact that $u(0,0) \neq 0$ and $\left.w(0,0)=0\right)$, we have

$$
\frac{\theta(t)-w(t, \theta(t))}{u(t, \theta(t))}=\frac{1}{\Gamma(p)} \int_{0}^{t} \frac{v(s)}{(t-s)^{1-p}} d s,
$$

i.e.

$$
\theta(t)=\frac{u(t, \theta(t))}{\Gamma(p)} \int_{0}^{t} \frac{v(s)}{(t-s)^{1-p}} d s+w(t, \theta(t)) .
$$

At this stage, our target is to prove the following existence theorem; see (1).

Theorem 2 Assume that the hypotheses (H1)-(H2) hold. Then the system (1) has a solution defined on $J$.

Proof Set $\mathfrak{s}=C(J, \mathbb{R})$ and a subset $S$ of $\mathfrak{s}$ defined by

$$
S=\{\theta \in \mathfrak{I} \mid\|\theta\| \leq N\}
$$

where $N=\frac{\Delta U_{0}+W_{0}}{1-\Delta\|\varphi\|-\|\psi\|}, U_{0}=\max _{t \in J}|u(t, 0)|, W_{0}=\max _{t \in J}|w(t, 0)|$, and $\Delta=\frac{\|v\|}{\Gamma(p+1)}$.

We take note of the fact that $S$ is a closed, convex, and bounded subset of the Banach algebra $\mathfrak{I}$. Now, we consider the system (1). Obviously, $\theta(t)$ is a solution of the FHDEs system (1) if and only if $\theta(t)$ satisfies the following system of integral equations:

$$
\left\{\begin{array}{l}
\theta(t)=\frac{u(t, \theta(t))}{\Gamma(p)} \int_{0}^{t} \frac{v(s, \vartheta(s))}{(t-s)^{1-p}} d s+w(t, \theta(s)), \\
\vartheta(t)=\frac{u(t, \vartheta(t))}{\Gamma(p)} \int_{0}^{t} \frac{v(s, \theta(s))}{(t-s)^{1-p}} d s+w(t, \vartheta(s)), \quad t \in J .
\end{array}\right.
$$

Let us define two operators $E, G: \mathfrak{I} \rightarrow \mathfrak{I}$ and $F: S \rightarrow \mathfrak{I}$ by

$$
\left\{\begin{array}{l}
E \theta(t)=u(t, \theta(t)) \\
F \theta(t)=\frac{1}{\Gamma(p)} \int_{0}^{t}(t-s)^{p-1} v(s, \theta(s)) d s, \quad t \in J \\
G \theta(t)=w(t, \theta(t))
\end{array}\right.
$$

so the system (3) is transformed into the system of operator equations

$$
\left\{\begin{array}{l}
\theta(t)=E \theta(t) F \vartheta(t)+G \theta(t), \\
\vartheta(t)=E \vartheta(t) F \theta(t)+G \vartheta(t), \quad t \in J .
\end{array}\right.
$$

We shall show that the operators $E, F$, and $G$ satisfy all the conditions of Theorem 2 .

Let $\theta, \vartheta \in \mathfrak{I}$, by the hypothesis (H1) we have

$$
\begin{aligned}
|E \theta(t)-E \vartheta(t)| & =|u(t, \theta(t))-u(t, \vartheta(t))| \\
& \leq \varphi(t)|\theta(t)-\vartheta(t)| \leq\|\varphi\|\|\theta-\vartheta\|,
\end{aligned}
$$

for all $t \in J$, which implies that $\|E \theta-E \vartheta\| \leq\|\varphi\|\|\theta-\vartheta\|$. Therefore, $E$ is a Lipschitzian on $\mathfrak{s}$ with Lipschitz constant $\|\varphi\|$. Analogously, $C$ is a Lipschitzian on $\mathfrak{\Im}$ with Lipschitz constant $\|\psi\|$. Next, we prove that $F$ is a compact and continuous operator on $S$. 
Let $\left\{\theta_{n}\right\}$ be a sequence in $S$ converging to a point $\theta \in S$. Then

$$
\begin{aligned}
\lim _{n \rightarrow \infty} F \theta_{n}(t) & =\frac{1}{\Gamma(p)} \lim _{n \rightarrow \infty}\left(\int_{0}^{t}(t-s)^{p-1} v(s, \theta(s)) d s\right) \\
& =\frac{1}{\Gamma(p)} \int_{0}^{t}(t-s)^{p-1} \lim _{n \rightarrow \infty} v\left(s, \theta_{n}(s)\right) d s \\
& =\frac{1}{\Gamma(p)} \int_{0}^{t}(t-s)^{p-1} v(s, \theta(s)) d s \\
& =F \theta(t),
\end{aligned}
$$

for all $t \in J$, where the second equality holds by the Lebesgue dominated convergence theorem. So $F$ is a continuous function on $S$.

Let $\theta \in S$, by assumption (H2), for $t \in J$, we have

$$
\begin{aligned}
|F \theta(t)| & =\frac{1}{\Gamma(p)}\left|\int_{0}^{t}(t-s)^{p-1} v(s, \theta(s)) d s\right| \\
& \leq \frac{1}{\Gamma(p)} \int_{0}^{t}(t-s)^{p-1}|v(s, \theta(s))| d s \\
& \leq \frac{1}{\Gamma(p)} \int_{0}^{t}(t-s)^{p-1} v(s) d s \\
& \leq \frac{\|v\|}{\Gamma(p)} \int_{0}^{t}(t-s)^{p-1} d s \\
& \leq \frac{\|v\|}{\Gamma(p+1)},
\end{aligned}
$$

taking the supremum over $t$, we obtain

$$
\|F \theta\| \leq \frac{\|v\|}{\Gamma(p+1)}=\Delta
$$

for all $\theta \in S$, so $F$ is uniformly bounded on $S$. Now letting $t_{1}, t_{2} \in J$, for any $\theta \in S$, one has

$$
\begin{aligned}
\left|F \theta\left(t_{1}\right)-F \theta\left(t_{2}\right)\right|= & \frac{1}{\Gamma(p)}\left|\int_{0}^{t_{1}}\left(t_{1}-s\right)^{p-1} v(s, \theta(s))-\int_{0}^{t_{2}}\left(t_{2}-s\right)^{p-1} v(s, \theta(s)) d s\right| \\
\leq & \frac{1}{\Gamma(p)}\left|\int_{0}^{t_{1}}\left(t_{1}-s\right)^{p-1} v(s, \theta(s)) d s-\int_{0}^{t_{1}}\left(t_{2}-s\right)^{p-1} v(s, x(s)) d s\right| \\
& +\frac{1}{\Gamma(p)}\left|\int_{0}^{t_{1}}\left(t_{2}-s\right)^{p-1} v(s, \theta(s)) d s-\int_{0}^{t_{2}}\left(t_{2}-s\right)^{p-1} v(s, \theta(s)) d s\right| \\
\leq & \frac{\|v\|}{\Gamma(p)}\left(\left|\int_{0}^{t_{1}}\left(t_{1}-s\right)^{p-1}-\left(t_{2}-s\right)^{p-1} d s\right|+\left|\int_{t_{1}}^{t_{2}}\left(t_{2}-s\right)^{p-1} d s\right|\right) \\
\leq & \Delta\left(\left|t_{1}^{p}-t_{2}^{p}\right|+\left|\left(t_{2}-t_{1}\right)^{p}\right|\right) .
\end{aligned}
$$

Since $t^{p}$ is uniformly continuous on $J$ for $0<p<1$, for any $\varepsilon>0$ there exists $\delta_{1}>0$ such that if $\left|t_{1}-t_{2}\right|<\delta_{1}$ we have

$$
\left|t_{1}^{p}-t_{2}^{p}\right|<\frac{1}{2 \Delta} \varepsilon
$$


Letting $\delta=\min \left\{\delta_{1},\left(\frac{1}{2 \Delta} \varepsilon\right)^{\frac{1}{p}}\right\}$, if $\left|t_{2}-t_{1}\right|<\delta$, we have

$$
\left|F \theta\left(t_{1}\right)-F \theta\left(t_{2}\right)\right|<\Delta\left(\frac{1}{2 \Delta} \varepsilon+\frac{1}{2 \Delta} \varepsilon\right)=\varepsilon .
$$

This implies that $F(S)$ is equi-continuous. As a result $F$ is completely continuous on $S$. In order to prove the hypothesis (C3) of Theorem 1 , let $\theta \in \Im$ and $\vartheta \in S$ such that $\theta=$ $E \theta F \vartheta+G \theta$; by assumptions (H1) and (H2) we have

$$
\begin{aligned}
|\theta(t)|= & |E \theta(t)||F \vartheta(t)|+|G \theta(t)| \\
\leq & \frac{\|v\|}{\Gamma(p)}(|u(t, \theta(t))-u(t, 0)|+|u(t, 0)|) \int_{0}^{t}|(t-s)|^{p-1} d s \\
& +(|w(t, \theta(t))-w(t, 0)|+|w(t, 0)|) \\
\leq & \frac{\|v\|}{\Gamma(p+1)}(\varphi(t)(|\theta(t)|)+|u(t, 0)|)+(\psi(t)(|\theta(t)|)+|w(t, 0)|) \\
\leq & \Delta\left(\|\varphi\|(\|\theta\|)+U_{0}\right)+\left(\|\psi\|(\|\theta\|)+W_{0}\right),
\end{aligned}
$$

then by taking the supremum over $t$ on $J$ and by taking into account (H2) we conclude that

$$
\|\theta\| \leq \frac{\Delta U_{0}+W_{0}}{1-\Delta\|\varphi\|-\|\psi\|}=N
$$

which implies that $\theta \in S$. Finally, we have

$$
4 \sigma\|F S\|+\delta \leq 4\|\varphi\| \Delta+\|\psi\|<1 .
$$

So, the assumption (C4) of Theorem 1 has been proved. Therefore, all the conditions of Theorem 1 are satisfied, hence the operator $T(\theta, \vartheta)=E \theta F \vartheta+G \vartheta$ has a coupled fixed point on $\widetilde{S}$. As a result, the FHDEs system (1) has a solution defined on $J$.

\section{Example}

Example 3 We consider the following system of fractional hybrid differential equations:

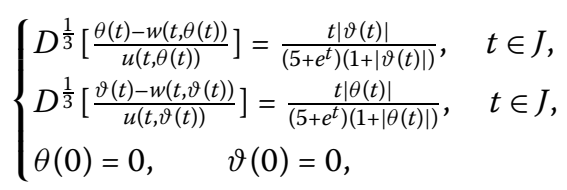

where

$$
\begin{aligned}
& u(t, \theta(t))=\frac{1}{17} t\left(\frac{1}{16} \cos \theta(t)+\frac{\theta^{2}(t)+|\theta(t)|}{1+|\theta(t)|}\right)+\frac{1}{8}, \\
& w(t, \theta(t))=\frac{1}{18} e^{1-t}\left(\frac{1}{4} \sin \theta(t)+\frac{\theta^{2}(t)+4|\theta(t)|}{2+|\theta(t)|}\right),
\end{aligned}
$$

for arbitrary $\theta, \vartheta \in \Im$ and $t \in J$, we can obtain

$$
|u(t, \theta(t))-u(t, \vartheta(t))| \leq \frac{t}{16}|\theta(t)-\vartheta(t)|,
$$




$$
\begin{aligned}
& |w(t, \theta(t))-w(t, \vartheta(t))| \leq \frac{e^{1-t}}{8}|\theta(t)-\vartheta(t)|, \\
& |v(t, \theta(t))-v(t, \vartheta(t))| \leq \frac{t}{\left(5+e^{t}\right)} .
\end{aligned}
$$

We conclude that $\varphi(t)=\frac{1}{16} t, \psi(t)=\frac{1}{8} e^{1-t},\|\varphi\| \approx 0.063,\|\psi\| \approx 0.34, F_{0} \approx 0.19, W_{0}=0$, $\Delta \approx 0.079, N \approx 0.023$. So

$$
4\|\varphi\| \Delta+\|\psi\| \approx 0.36<1
$$

\section{As a result it follows by Theorem 2 that the problem (4) has a solution.}

\section{Competing interests}

The authors declare that they have no competing interests.

\section{Authors' contributions}

All authors contributed equally and significantly in writing this article. All authors read and approved the final manuscript.

\section{Author details}

${ }^{1}$ Department of Mathematics and Computer Science, Amirkabir University of Technology, 424 Hafez Ave, Tehran, Iran.

${ }^{2}$ Research Institute for Natural Sciences, Hanyang University, Seoul, 133-791, Korea.

\section{Acknowledgements}

The authors are grateful to the reviewers for their valuable comments and suggestions.

\section{Received: 2 November 2015 Accepted: 16 February 2016 Published online: 24 February 2016}

\section{References}

1. Dhage, BC: A fixed point theorem in Banach algebras involving three operators with applications. Kyungpook Math. J. 44, 145-155 (2004)

2. Dhage, BC: Quadratic perturbations of periodic boundary value problems of second order ordinary differential equations. Differ. Equ. Appl. 2, 465-486 (2010)

3. Dhage, BC: Nonlinear quadratic first order functional integro-differential equations with periodic boundary conditions. Dyn. Syst. Appl. 18, 303-322 (2009)

4. Dhage, BC, Karande, BD: First order integro-differential equations in Banach algebras involving Caratheodory and discontinuous nonlinearities. Electron. J. Qual. Theory Differ. Equ. 2005, 21 (2005) 16 pp.

5. Dhage, BC, O'Regan, BD: A fixed point theorem in Banach algebras with applications to functional integral equations Funct. Differ. Equ. 7, 259-267 (2000)

6. Dhage, BC, Salunkhe, SN, Agarwal, RP, Zhang, W: A functional differential equation in Banach algebras. Math. Inequal. Appl. 8, 89-99 (2005)

7. Dhage, BC: On $\alpha$-condensing mappings in Banach algebras. Math. Stud. 63, 146-152 (1994)

8. Dhage, BC, Lakshmikantham, V: Basic results on hybrid differential equations. Nonlinear Anal. Hybrid Syst. 4, 414-424 (2010)

9. Dhage, BC: A nonlinear alternative in Banach algebras with applications to functional differential equations Nonlinear Funct. Anal. Appl. 8, 563-575 (2004)

10. Dhage, BC: Fixed point theorems in ordered Banach algebras and applications. Panam. Math. J. 9, 93-102 (1999)

11. Caputo, M: Linear models of dissipation whose Q is almost independent, II. Geophys. J. R. Astron. Soc. 13, 529-539 (1967)

12. Diethelm, K, Ford, NJ: Analysis of fractional differential equations. J. Math. Anal. Appl. 265, 229-248 (2002)

13. Diethelm, K, Ford, NJ: Multi-order fractional differential equations and their numerical solution. Appl. Math. Comput. $154,621-640$ (2004)

14. El-Sayed, AMA, Ibrahim, AG: Set-valued integral equations of fractional-orders. Appl. Math. Comput. 118, 113-121 (2001)

15. Gafiychuk, V, Datsun, B, Meleshko, V: Mathematical modeling of time fractional reaction diffusion systems. J. Comput. Appl. Math. 220, 215-225 (2008)

16. He, JH: Approximate analytical solution for seepage flow with fractional derivatives in porous media. Comput. Methods Appl. Mech. Eng. 167, 57-68 (1998)

17. Jumarie, G: An approach via fractional analysis to non-linearity induced by coarse-graining in space. Nonlinear Anal., Real World Appl. 11, 535-546 (2010)

18. Qiu, T, Bai, ZH: Positive solutions for boundary value problem of nonlinear fractional differential equation. J. Nonlinear Sci. Appl. 2008, 123-131 (2008)

19. Alomari, AK: A novel solution for fractional chaotic Chen system. J. Nonlinear Sci. Appl. 2015, 478-488 (2015)

20. Zhao, Y, Sun, S, Han, Z, Li, Q: Theory of fractional hybrid differential equations. Comput. Math. Appl. 62, 1312-1324 (2011)

21. Sitho, S, Ntouyas, SK, Tariboon, J: Existence results for hybrid fractional integro-differential equations. Bound. Value Probl. 2015, 113 (2015) 
22. Bai, C, Fang, J: The existence of a positive solution for a singular coupled system of nonlinear fractional differential equations. Appl. Math. Comput. 150, 611-621 (2004)

23. Chen, Y, An, H: Numerical solutions of coupled Burgers equations with time and space fractional derivatives. Appl. Math. Comput. 200, 87-95 (2008)

24. Gejji, VD: Positive solutions of a system of non-autonomous fractional differential equations. J. Math. Anal. Appl. 302, 56-64 (2005)

25. Lazarevic, MP: Finite time stability analysis of $\mathrm{PD}^{\alpha}$ fractional control of robotic time-delay systems. Mech. Res. Commun. 33, 269-279 (2006)

26. Su, X: Boundary value problem for a coupled system of nonlinear fractional differential equations. Appl. Math. Lett. $22,64-69$ (2009)

27. Gafiychuk, V, Datsko, B, Meleshko, V, Blackmore, D: Analysis of the solutions of coupled nonlinear fractional reaction-diffusion equations. Chaos Solitons Fractals 41, 1095-1104 (2009)

28. Ahmad, B, Ntouyas, SK, Alsaedi, A: Existence results for a system of coupled hybrid fractional differential equations. Sci. World J. 2014, 426-438 (2014)

29. Kilbas, AA, Srivastava, HM, Trujillo, JJ: Theory and Applications of Fractional Differential Equations. Elsevier, Amsterdam (2006)

30. Podlubny, I: Fractional Differential Equations. Academic Press, New York (1999)

31. Chang, SS, Cho, YJ, Huang, NJ: Coupled fixed point theorems with applications. J. Korean Math. Soc. 33, 575-585 (1996)

\section{Submit your manuscript to a SpringerOpen ${ }^{\circ}$ journal and benefit from:}

- Convenient online submission

- Rigorous peer review

- Immediate publication on acceptance

- Open access: articles freely available online

- High visibility within the field

Retaining the copyright to your article 